\title{
Escala de Satisfação dos Pacientes com os Serviços de Saúde Mental (SATIS-BR): estudo de validação
}

\author{
Patients' Satisfaction with Mental Health Services \\ Scale (SATIS-BR): validation study \\ Marina Bandeira', Mônia Aparecida da Silva²
}

\section{RESUMO}

Objetivo: Fazer um estudo da validade de construto, validade convergente e consistência interna da Escala de Satisfação dos Pacientes com os Serviços de Saúde Mental (SATIS-BR). Método: Participaram da pesquisa 110 pacientes psiquiátricos, atendidos em cinco serviços públicos de saúde mental do interior de Minas Gerais. A escala foi aplicada em entrevistas individuais estruturadas. A escala possui 12 itens que avaliam a satisfação dos pacientes, com alternativas de resposta dispostas em escala Likert de 5 pontos. Foi também aplicada a Escala de Mudança Percebida (EMP) para a análise de validade convergente da SATIS-BR. Resultados: A análise fatorial pelo método Principal Axis Factoring revelou uma estrutura fatorial de três fatores, que avaliam a satisfação dos pacientes em relação às dimensões: 1. Competência e compreensão da equipe; 2 . Ajuda e acolhida; 3 . Condições físicas do ser-

\section{Palavras-chave}

Satisfação do paciente, escalas, avaliação de resultados (cuidados de saúde), serviços de saúde mental. viço. A análise pelo coeficiente alfa de Cronbach mostrou boa consistência interna (alfa = 0,88). A análise da validade convergente foi adequada, tendo sido obtida uma correlação de Pearson positiva significativa com a escala EMP, que avalia um construto teoricamente relacionado ao de satisfação $(r=0,41 ; p<0,001)$. Conclusão: A escala SATIS-BR apresenta qualidades psicométricas adequadas de validade de construto, validade convergente e fidedignidade.

\section{ABSTRACT}

Objective: To investigate the construct validity, convergent validity and reliability of the Patient Satisfaction with Mental Health Services Scale (SATIS-BR). Method: One hundred and ten psychiatric patients attending five public mental health services located in Minas Gerais, Brazil, participated in this research. The scale was applied in individual structured interviews. The scale has 12 items evaluating patients' satisfaction, with response alternatives disposed in a five points Likert scale. In order to evaluate the convergent validity, another scale was applied, the Perceived Improvement Questionnaire (PIQ). Results: Factorial analysis using the Principal Axis Factoring method revealed a 3 factors structure, evaluating satisfaction regarding the following dimensions: 1. Professional competence

\footnotetext{
1 Universidade Federal de São João Del Rei (UFSJ); Conselho Nacional de Desenvolvimento Científico e Tecnológico (CNPq); Université de Montréal, Canadá.

2 Programa de Mestrado em Psicologia da UFSJ; Laboratório de Pesquisa em Saúde Mental (LAPSAM) da UFSJ.
}

Endereço para correspondência: Marina Bandeira

Rua Antonia da Encarnação Xavier, 120

36325-000 - Tiradentes, MG

Telefone: (32) 3379-2469

Telefax: (32) 33792457

E-mails: marina@mgconecta.com.br e bandeira@ufsj.edu.br 


\section{Keywords}

Patient satisfaction, scales, outcome assessment (health care), mental health services. and understanding; 2. Help from the professional; 3. Service physical conditions. Reliability results showed adequate internal consistency evaluated by Cronbach analysis (alpha $=0,88$ ). Convergent validity analysis showed a positive significant Pearson Correlation coefficient $(r=$ 0,$41 ; p<0,001$ ) with the PIQ scale, which evaluates the related construct of patient" perceived improvement. Conclusion: The SATIS-BR scale has adequate psychometric properties of construct validity, convergent validity and reliability.

\section{INTRODUÇÃO}

A avaliação contínua dos serviços de saúde mental tem sido recomendada, visando promover a qualidade desses serviços ${ }^{1}$. Segundo Donabedian², os resultados da assistência em saúde devem estar congruentes com a perspectiva dos usuários, para que a qualidade do tratamento não fique comprometida. Incluir a perspectiva dos próprios pacientes na avaliação dos serviços de saúde mental tem despertado cada vez mais o interesse dos clínicos, dos administradores e das agências de credenciamento de serviços ${ }^{3}$. Uma das medidas mais frequentemente utilizadas para avaliar a qualidade dos serviços é a satisfação dos pacientes ${ }^{4}$. Dando destaque à importância da perspectiva dos pacientes, Ruggeri ${ }^{5}$ apontou que a medida subjetiva de satisfação dos pacientes com os serviços constitui o critério, por excelência, da qualidade dos serviços.

A avaliação da satisfação dos pacientes é importante, porque está relacionada com maior frequência de utilização dos serviços e diminuição da taxa de abandono do tratamento ${ }^{6-8}$, além de ser um fator preditivo para menor taxa de hospitalizações futuras ${ }^{9}$. A avaliação da satisfação dos pacientes é importante também por causa de sua associação com a adesão ao tratamento ${ }^{7}$. A adesão é essencial para a obtenção de resultados positivos das intervenções, particularmente em saúde mental, em que há dificuldade dos pacientes psiquiátricos em aceitar e manter o tratamento medicamentoso ${ }^{10}$. A inclusão da avaliação dos pacientes pode contribuir, ainda, para aumentar sua autoestima e o sentimento de controle ou empoderamento (empowerment), ao perceber que seu ponto de vista está sendo considerado ${ }^{11}$.

Entretanto, cuidados metodológicos devem ser tomados para a avaliação da satisfação dos pacientes com os serviços, tanto em termos da validade interna dos delineamentos de pesquisa e da representatividade da amostra avaliada quanto das qualidades psicométricas dos instrumentos de medida usados para aferir a satisfação ${ }^{4-6,12}$. Os instrumentos de medida devem apresentar as propriedades de validade e fidedignidade e ser multidimensionais, ou seja, avaliar diferentes dimensões do fenômeno, de preferência com subescalas que forneçam escores independentes para cada dimensão. As medidas multidimensionais são mais sensíveis aos efeitos do tratamento nos serviços de saúde mental, uma vez que podem ocorrer avaliações favoráveis a algumas dessas dimensões, e não a outras $5^{5,6,12-15}$. Esse tipo de medida permite, portanto, que os profissionais identifiquem os aspectos do serviço menos apreciados pelos pacientes e realizem modificações visando melhorar sua qualidade ${ }^{16}$. Em uma análise de estudos que avaliaram a satisfação dos usuários, Ruggeri ${ }^{5}$ destacou que as escalas de medida unidimensionais ou baseadas em um único item global não são consideradas adequadas para avaliar os resultados dos serviços, mas sim os instrumentos multidimensionais, pois o construto satisfação é multidimensional.

Para avaliar a percepção dos pacientes sobre os serviços de saúde mental no Brasil, a Escala da Satisfação dos Pacientes com os Serviços de Saúde Mental (SATIS-BR) havia sido submetida a um estudo de adaptação semântica e cultural para o país, a partir da versão canadense da escala, assim como uma avaliação de sua fidedignidade e validade concomitante ${ }^{17,18}$. Entretanto, ainda não havia sido realizada a análise da validade de construto, por meio de uma análise fatorial, visando identificar a estrutura dimensional da escala, para o contexto brasileiro. A escala original canadense tinha apresentado uma estrutura dimensional com três fatores que explicaram 54\% da variância dos dados e uma consistência interna adequada, com alfa de Cronbach igual a 0,89. Essa escala é específica para essa população-alvo e não foi adaptada para outros tipos de população. A presente pesquisa teve como objetivo completar as análises anteriores da escala brasileira, realizando um estudo da análise fatorial, assim como reavaliar sua consistência interna e avaliar sua validade convergente.

\section{MÉTODO}

\section{Participantes}

Participaram desta pesquisa 110 pacientes atendidos em cinco serviços públicos de saúde mental de três cidades do interior de Minas Gerais. A distribuição dos pacientes por cidade foi a seguinte: 40,00\%, 33,60\% e 26,40\%. Foram incluídos, na amostra, pacientes psiquiátricos que estavam em tratamento há, pelo menos, um ano no serviço. Foram adotados, como critérios de exclusão: o paciente estar em crise, possuir comorbidade de déficits cognitivos associado ao problema psiquiátrico e apresentar dificuldade de responder às questões da escala. Foram contatados 143 pacientes para participar da pesquisa, mas 33 foram excluídos por estarem em cri- 
se, apresentarem comorbidades de transtornos por déficits cognitivos ou por não terem compreendido as questões dos instrumentos utilizados. Entretanto, não temos os dados que identifiquem quantos foram excluídos por um ou outro motivo. Não houve recusas dos pacientes em participar do estudo. Entretanto, a amostragem foi não probabilística, tendo sido selecionados os pacientes disponíveis nos serviços que preenchiam os critérios de inclusão e exclusão. A presença de comorbidade foi verificada por meio de consulta aos prontuários. O tamanho da amostra utilizada se situa dentro de uma faixa considerada aceitável para a realização da análise fatorial, embora não haja consenso sobre essa questão, na área $^{19}$. Os valores de adequação da amostra obtidos foram satisfatórios $(\mathrm{KMO}=0,87$; Bartlet $=446,507 ; p<0,01)$.

\section{Instrumentos de medida}

\section{Escala de Satisfação dos Pacientes com os Serviços de} Saúde Mental (SATIS-BR): Este instrumento foi elaborado originalmente pela Organização Mundial de Saúde (OMS), como parte do projeto WHO-SATIS21, que visava construir instrumentos de avaliação dos serviços de saúde mental, que incluíssem a satisfação dos três agentes envolvidos nos serviços - pacientes, familiares e profissionais -, assim como a sobrecarga sentida pelos profissionais e familiares. Sua adaptação e sua validação para o Brasil foram feitas por Bandeira et al. ${ }^{17}$ e Bandeira et al. ${ }^{18}$, com base na escala canadense, validada previamente. A escala inicial brasileira era composta por 13 questões, com alternativas de resposta dispostas em uma escala Likert de 5 pontos, na qual o valor 1 indica o menor grau de satisfação e o valor 5 indica o maior grau de satisfação com o serviço. A escala propriamente dita faz parte de um questionário mais completo contendo 44 questões, que incluem itens quantitativos, qualitativos, descritivos e sociodemográficos, descritos nos artigos citados acima. O questionário completo assim como a escala propriamente dita estão disponíveis no site do Laboratório de Pesquisa em Saúde Mental (LAPSAM) da Universidade Federal de São João del Rei (UFSJ).

No estudo brasileiro, a escala SATIS-BR tinha sido submetida a uma análise de sua consistência interna, por meio do cálculo de alfa de Cronbach, para a escala global e para as subescalas que estavam presentes na escala canadense. Os resultados indicaram índices adequados de alfa de Cronbach para a escala global $(0,84)$ e para as duas subescalas que tinham sido retidas no estudo brasileiro, após a análise efetuada $(0,77$ e 0,55). Foi feita igualmente uma análise das correlações entre essas subescalas, tendo sido obtidas correlações positivas e significativas entre elas $(0,62)$ e entre cada uma delas e a escala global $(0,87$ e 0,88$)$. A escala tinha apresentado igualmente uma correlação positiva e significativa $(0,63)$ com a escala CSQ-8 de satisfação dos pacientes com serviços de saúde mental22. Entretanto, não tinha sido realizada a análise fatorial para a versão brasileira da escala, tendo em vista o número pequeno de sujeitos da amostra do estudo $(N=30)$. Esse número reduzido de sujeitos consiste em uma limitação do estudo anterior, razão pela qual o presente estudo visou refazer as análises citadas acima, além de realizar a Análise Fatorial. Portanto, não tinha sido identificada a estrutura dimensional da escala, ou seja, a distribuição dos seus itens entre os fatores, para a versão brasileira ${ }^{18}$, objeto do presente trabalho.

Escala de Mudança Percebida (EMP): A versão original desta escala, "Questionaire of Perceived Changes", foi elaborada por Mercier et al. ${ }^{23}$ no Canadá visando avaliar a percepção de mudanças pelos pacientes como resultado do tratamento recebido em serviços de saúde mental. Posteriormente, a escala foi reavaliada por Perreault et al. ${ }^{24}$, com a denominação de Perception of Improvement Questionnaire (PIQ). Esse instrumento foi submetido aos procedimentos de adaptação transcultural para o Brasil ${ }^{25}$ e foi validado por Bandeira et al. ${ }^{26}$. A versão brasileira da escala EMP contém 19 itens, que avaliam a percepção do paciente sobre as mudanças ocorridas em sua vida, como resultado do tratamento recebido, com alternativas de resposta dispostas em uma escala Likert de 3 pontos, sendo 1. Pior do que antes, 2. Sem mudança, 3. Melhor do que antes. Dentre os 19 itens da escala, um item global avalia como o paciente percebe, em geral, os efeitos do tratamento recebido, enquanto os demais itens avaliam as mudanças percebidas em diversas dimensões de sua vida, incluindo aspectos físicos, psicológicos, vida social, condições de vida etc. ${ }^{26}$.

Uma análise da estrutura fatorial da escala EMP, pelo método dos Componentes Principais, com rotação Varimax, resultou em estrutura de três fatores: 1. Ocupação e saúde física; 2. Aspectos psicológicos e sono; 3. Relacionamentos e estabilidade emocional. A estrutura fatorial com três fatores, com valores de eigenvalues acima de 1,0, explicou 42,13\% da variância dos dados, tendo todos os itens cargas fatoriais acima de 0,47. A escala apresentou boa consistência interna (alfa de Cronbach $=0,85$ ), estabilidade temporal teste-reteste $(r=0,93 ; p<0,05)$ e validade convergente $(r=0,37 ; p<0,05)$. Os indicadores obtidos de adequação da amostra foram: $\mathrm{KMO}=0,88$; Bartlet $=1216,90 ; p<0,01^{26}$.

Questionário sociodemográfico e clínico: Foi utilizado um questionário visando avaliar as características sociodemográficas e clínicas dos pacientes. Este questionário havia sido previamente testado em um estudo piloto, visando avaliar a adequação das questões para um bom entendimento pela população-alvo. As variáveis sociodemográficas incluídas no questionário foram: idade, sexo, escolaridade e renda. As variáveis clínicas avaliadas foram: diagnóstico, idade em que ficou doente, há quantos anos estava doente, tempo de tratamento, existência de outra doença além do transtorno psiquiátrico, dados sobre internações em hospital psiquiátriCo, tipo e frequência da medicação, dados sobre a ocorrência de crises no ano anterior, uso de bebidas ou de drogas. 
Algumas variáveis desse questionário foram identificadas por meio de consulta aos prontuários dos serviços de saúde mental, tais como o diagnóstico dos pacientes e a presença de comorbidades.

\section{Procedimento}

Coleta de dados: Após aprovação do projeto pelos serviços de saúde mental e pela Comissão de ética da UFSJ (processo 8-a/2010/CEPES), os participantes foram contatados para participar da pesquisa. As entrevistas foram realizadas na própria instituição ou na casa do entrevistado, conforme sua disponibilidade. Antes do início de cada entrevista, eram explicados ao paciente os objetivos da pesquisa, o procedimento, o anonimato dos dados e sua liberdade de interromper a qualquer momento a realização da entrevista se desejasse desistir da sua participação. Era explicado, ainda, que não havia respostas certas ou erradas e que ele deveria apenas responder, de forma sincera, de acordo com a sua opinião. Durante as entrevistas, os entrevistadores aplicavam as escalas EMP e SATIS-BR, assim como o questionário sociodemográfico, lendo as questões para os participantes e marcando suas respostas.

Análise de dados: $O$ procedimento adotado para verificação da validade de construto da escala SATIS-BR foi a análise fatorial, pelo Método dos Eixos Principais, com rotação Varimax. Foi utilizado o critério mínimo de 0,40 de carga fatorial para retenção dos itens na escala ${ }^{27}$. O critério para delimitação do número de fatores a serem retidos foi definido com base nos autovalores superiores a 1.0 e análise gráfica dos autovalores ${ }^{28}$.

O segundo procedimento realizado foi a Análise por Hipóteses, enfocando a técnica de validação convergente. Esse procedimento visa verificar a correlação da escala com uma outra que avalia um construto distinto, porém teoricamente relacionado ${ }^{29}$. Para isso, foi utilizado o construto de percepção de mudança pelos próprios pacientes, pois, no estudo realizado por Holcomb et al. ${ }^{3}$, esses autores constataram que a melhora percebida pelos pacientes entre o pré e o pós-teste era um importante preditor do sentimento posterior de satisfação com os serviços. Perreault et al..$^{24}$ também encontraram uma relação significativa entre esses dois construtos. Tendo em vista essa relação observada entre percepção de mudança e satisfação com o serviço, foi escolhida a Escala de Percepção de Mudança (EMP) para avaliar a validade convergente da escala SATIS-BR. A análise dos dados foi feita por meio do cálculo da correlação de Pearson entre os escores dessas duas escalas de medida.

A fidedignidade da escala SATIS-BR foi avaliada por meio da análise de sua consistência interna, por meio do coeficiente alfa de Cronbach. Foi utilizado como critério mínimo para retenção dos itens na escala o valor de 0,20 de correlação item-tota ${ }^{30}$. Para a realização dessas análises estatísticas, foi utilizado o programa estatístico SPSS-PC, versão 13.0.

\section{RESULTADOS}

\section{Descrição da amostra}

As características sociodemográficas da amostra estão apresentadas na tabela 1. Os pacientes eram, em sua maioria, do sexo feminino $(68,20 \%)$, solteiros $(38,20 \%)$ e alfabetizados (97,30\%), com escolaridade predominante de ensino fundamental incompleto (65,60\%). A idade média era de 42,11 anos ( $d p=10,77)$, sendo a mínima de 19 e a máxima de 67 anos. Grande parte possuía renda própria (59,10\%), cujo valor era, na maioria dos casos (78,50\%), de 1 a 2 salários mínimos e as principais fontes de renda eram o auxílio do governo $(21,60 \%)$ e a aposentadoria por invalidez $(21,60 \%)$.

Tabela 1. Características sociodemográficas dos pacientes

\begin{tabular}{|c|c|c|c|}
\hline Variáveis & Categorias & Frequências (n) & Porcentagens \\
\hline \multirow[t]{2}{*}{ Sexo } & Feminino & 75 & 68,20 \\
\hline & Masculino & 35 & 31,80 \\
\hline \multirow[t]{6}{*}{ Estado civil } & Solteiro & 42 & 38,20 \\
\hline & Casado & 39 & 35,40 \\
\hline & Separado ou divorciado & 14 & 12,70 \\
\hline & Vivendo como casado & 6 & 5,50 \\
\hline & Viúvo & 6 & 5,50 \\
\hline & Não informaram & 3 & 2,70 \\
\hline \multirow{3}{*}{$\begin{array}{l}\text { Local em que } \\
\text { faz } \\
\text { tratamento }\end{array}$} & CAPS de Lavras & 44 & 40,00 \\
\hline & CAPS Del Rei & 37 & 33,60 \\
\hline & Ambulatório de Barbacena & 29 & 26,40 \\
\hline \multirow{7}{*}{$\begin{array}{l}\text { Nível de } \\
\text { escolaridade }\end{array}$} & Analfabeto & 3 & 2,70 \\
\hline & Ensino fundamental incompleto & 72 & 65,60 \\
\hline & Ensino fundamental completo & 13 & 11,80 \\
\hline & Ensino médio incompleto & 5 & 4,50 \\
\hline & Ensino médio completo & 15 & 13,60 \\
\hline & Superior completo & 1 & 0,90 \\
\hline & Curso técnico & 1 & 0,90 \\
\hline \multirow{2}{*}{$\begin{array}{l}\text { Possui renda } \\
\text { própria }\end{array}$} & Sim & 65 & 59,10 \\
\hline & Não & 45 & $40,90 \%$ \\
\hline \multirow[t]{4}{*}{ Renda } & Menos de 1 salário mínimo & 7 & 10,70 \\
\hline & De 1 a 2 salários mínimos & 51 & 78,50 \\
\hline & De 2,1 a 3 salários mínimos & 5 & 7,70 \\
\hline & Não informaram & 2 & 3,10 \\
\hline \multirow{11}{*}{$\begin{array}{l}\text { Origem da } \\
\text { renda }\end{array}$} & Emprego ou trabalho regular & 13 & 20,00 \\
\hline & Trabalho temporário & 1 & 1,50 \\
\hline & Aposentadoria por idade & 1 & 1,50 \\
\hline & Aposentadoria por invalidez & 14 & 21,60 \\
\hline & Auxílio-doença & 5 & 7,70 \\
\hline & Seguro & 1 & 1,50 \\
\hline & Pensão & 10 & 15,40 \\
\hline & Emprego e pensão & 2 & 3,10 \\
\hline & Bolsas de Programas do Governo & 3 & 4,60 \\
\hline & Aluguel & 1 & 1,50 \\
\hline & Encostado (auxílio do governo) & 14 & 21,60 \\
\hline
\end{tabular}

As características clínicas da amostra estão apresentadas na tabela 2. Quarenta por cento dos pacientes tinham um diagnóstico da categoria Esquizofrenia, Transtornos Esquizotípicos e Transtornos Delirantes, conforme os critérios da CID-1020. A maioria dos pacientes $(86,40 \%)$ não apresentava comorbidades e não tinha doenças físicas (58,20\%). A idade de início do transtorno psiquiátrico foi, em média, de 28,48 anos $(\mathrm{dp}=12,09)$, variando de 1 a 64 , e a duração média do 
Tabela 2. Características clínicas dos pacientes

\begin{tabular}{|c|c|c|c|c|}
\hline Variáveis & Categorias & Frequência (n) & Porcentagem $(\%)$ & Média/DP \\
\hline \multirow[t]{3}{*}{ Categoria diagnóstica } & Esquizofrenia, Transtornos Esquizotípicos e Delirantes & 44 & $44(40,00 \%)$ & \\
\hline & Transtornos do Humor e Afetivos & 53 & $53(48,20 \%)$ & \\
\hline & Transtornos Neuróticos Relacionados ao Estresse e Somatoforme & 13 & $13(11,80 \%)$ & \\
\hline \multirow[t]{2}{*}{ Comorbidades } & Sim & 15 & $15(13,60 \%)$ & \\
\hline & Não & 95 & $95(86,40 \%)$ & \\
\hline \multirow[t]{5}{*}{ Crises no último ano } & Não teve crises & 59 & $59(53,60 \%)$ & 1,45 crises $\pm 2,99$ \\
\hline & Teve 1 crise & 27 & $27(24,60 \%)$ & \\
\hline & Teve de 2 a 5 crises & 17 & $17(15,50 \%)$ & \\
\hline & Teve de 6 a 10 crises & 5 & $5(4,50 \%)$ & \\
\hline & Teve 10 ou mais crises & 2 & $2(1,80 \%)$ & \\
\hline \multirow[t]{6}{*}{ Internações } & Nunca foi internado & 55 & $50,00 \%$ & 3,96 vezes $\pm 13,88$ \\
\hline & Teve de 1 a 5 internações & 39 & $35,60 \%$ & \\
\hline & Teve de 6 a 10 internações & 10 & $9,00 \%$ & \\
\hline & Teve de 11 a 15 internaçōes & 2 & $1,80 \%$ & \\
\hline & Teve mais de 16 internações & 2 & $1,80 \%$ & \\
\hline & Não souberam informar & 2 & $1,80 \%$ & \\
\hline \multirow[t]{3}{*}{ Tipo de medicação recebida } & Apenas via oral & 83 & $75,50 \%$ & \\
\hline & Apenas via intramuscular & 1 & $0,90 \%$ & \\
\hline & Ambas & 26 & $23,60 \%$ & \\
\hline \multirow[t]{2}{*}{ Doenças físicas } & Sim & 46 & $41,80 \%$ & \\
\hline & Não & 64 & $58,20 \%$ & \\
\hline
\end{tabular}

transtorno psiquiátrico de 13,66 anos ( $d p=10,83$ ), variando entre 1 e 46 anos. A duração média do tratamento psiquiátrico era de 11,05 anos (dp=9,69), com tempo mínimo de 1 e máximo de 46 anos. Quanto ao número de crises no último ano, 53,60\% dos pacientes não tinham sofrido nenhuma crise. Dos entrevistados, 50,00\% relataram que haviam sido internados em hospitais psiquiátricos, com uma média de 3,96 internações ( $d p=13,88)$, no decorrer da vida, variando de 1 a 30. A medicação era apenas do tipo oral para a maioria dos pacientes $(75,50 \%)$, com um número médio de 3,26 ( $\mathrm{dp}=$ 1.31) tipos de medicamentos, variando de 1 a 8.

\section{Dados da validação da escala}

A análise da estrutura fatorial pelo método de Principal Axis Factoring, com rotação Varimax, identificou três fatores (Tabela 3). Os indicadores obtidos de adequação da amostra foram: $\mathrm{KMO}=0,87$; Bartlet $=446,507 ; p<0,01$. A estrutura fatorial, com três fatores, com valores de eigenvalues acima de 1,0, explicou 53,12\% da variância dos dados, tendo todos os itens cargas fatoriais acima de 0,45.

Os três fatores ou subescalas identificados foram: 1. Competência e compreensão da equipe, 2. Ajuda e acolhida, 3. Condições físicas do serviço. O primeiro fator contém sete itens (itens 2, 3, 4, 5, 6, 9 e 10), que explicaram 25,05\% da variância dos dados e avaliam a competência e compreensão dos profissionais a respeito do problema do paciente. $\mathrm{O}$ segundo fator contém três itens (itens 1,7 e 8), que explicaram $14,16 \%$ da variância dos dados e avaliam a ajuda recebida pelo paciente no serviço e a qualidade da acolhida dos profissionais. O terceiro fator possui dois itens (itens 11 e 12), que explicaram 13,92\% da variância dos dados e avaliam as condições físicas e conforto do serviço. Apenas um item da escala inicial brasileira (item 6, que avaliava a informação recebida pelo paciente) foi eliminado por apresentar um coeficiente de saturação abaixo do critério mínimo estabelecido.

A tabela 4 apresenta as correlações de Pearson dos fatores entre si e com a escala global. Pode-se notar que todas as correlações foram significativas e positivas e que as correlações entre cada fator e a escala global $(0,73,0,75$ e 0,94) foram mais elevadas do que aquelas obtidas entre os fatores $(0,46$ a 0,53). Assim, embora os fatores representem dimensões distintas, todos estão relacionados a um construto comum de satisfação, presente na escala SATIS-BR ${ }^{14}$.

No que se refere à validade convergente da escala EMP, os resultados mostraram uma correlação de Pearson positiva significativa $(r=0,41 ; p<0,001)$ entre os escores obtidos com a aplicação da escala SATIS-BR e os dados encontrados com a escala EMP. Essa escala avalia um construto distinto, porém teoricamente relacionado, que se refere à mudança percebida pelos próprios pacientes como resultado do tratamento recebido.

A fidedignidade da escala SATIS-BR foi avaliada por meio da análise de sua consistência interna, pelo cálculo do coeficiente alfa de Cronbach. Os resultados estão apresentados na tabela 1. Os dados indicaram, para a escala global, um valor de alfa de 0,88 e correlações item-total entre 0,87 e 0,88. Esse valor de alfa se situa dentro da faixa considerada ideal $(0,75$ a $0,85)$ por Vallerand ${ }^{14}$, indicando que as questões da escala são homogêneas, sem ser repetitivas. O primeiro fator apresentou um valor de alfa igual a 0,85 e correlações item-total entre 0,52 e 0,70. O segundo fator apresentou um valor de alfa igual a 0,61 e correlações item-total entre 0,37 e 0,52. O terceiro fator apresentou um valor de alfa igual a 0,74 e correlações item-total de 0,59. A escala SATIS-BR se encontra no anexo deste artigo. 
Tabela 3. Coeficientes de saturação dos itens nos três fatores extraídos da Análise Fatorial e valores de alfa de Cronbach para cada fator da Escala de Satisfação dos Pacientes com os Serviços de Saúde Mental (SATIS-BR)

\begin{tabular}{|c|c|c|c|}
\hline Itens/Fatores & Competência e compreensão da equipe & Acolhida e ajuda & Condiç̧̄es fisicas e conforto do serviço \\
\hline 6. Discussão sobre o tratamento & 0,69 & & \\
\hline 4. Equipe compreendeu ajuda necessária & 0,68 & & \\
\hline 2. Foi ouvido na admissão & 0,65 & & \\
\hline 3. Compreensão do problema na admissão & 0,62 & & \\
\hline 10. Competência do terapeuta principal & 0,61 & & \\
\hline 9. Competência da equipe & 0,59 & & \\
\hline 5. Tipo de ajuda recebida no serviço & 0,45 & & \\
\hline 8. Acolhida dos profissionais & & 0,62 & \\
\hline 7. Ajuda da equipe & & 0,57 & \\
\hline 1. Maneira de ser tratado (respeito e dignidade) & & 0,53 & \\
\hline 11. Conforto e aparência do serviço & & & 0,79 \\
\hline 12. Condições das instalações & & & 0,73 \\
\hline Valores de alfa de Cronbach & $(a=0,85)$ & $(a=0,61)$ & $(a=0,74)$ \\
\hline
\end{tabular}

KMO medida de adequaçăo da amostra (KMO $=0,88)$.

Teste de Bartlet: qui quadrado $=446,509 p<0,01$.

Variância explicada $=53,12 \%$.

Tabela 4. Correlações entre os fatores e a escala global da SATIS-BR

\begin{tabular}{lccc}
\hline Fatores & Fator 1 & Fator 2 & Fator 3 \\
\hline Fator 1 & 1 & $0,58^{*}$ & $0,52^{*}$ \\
Fator 2 & $0,58^{*}$ & 1 & $0,46^{*}$ \\
Fator 3 & $0,52^{*}$ & $0,46^{*}$ & 1 \\
Escala global & $0,94^{*}$ & $0,75^{*}$ & $0,73^{*}$ \\
\hline${ }^{*} p<0,01$. & & &
\end{tabular}

\section{DISCUSSÃO}

Os resultados obtidos indicaram que a escala SATIS-BR apresenta qualidades psicométricas adequadas, com relação à sua validade de construto, à validade convergente e à sua consistência interna. Na avaliação da validade de construto, a análise fatorial da escala indicou uma matriz com extração de três fatores, com todos os itens alcançando cargas fatoriais acima de 0,45, explicando 53,12\% da variância dos dados. Esses resultados se assemelham aos dados obtidos na validação da escala original canadense ${ }^{31}$, na qual também foram encontrados três fatores, explicando uma porcentagem comparável da variância dos dados (54,10\%).

Os três fatores identificados na versão brasileira da escala SATIS-BR são semelhantes, quanto ao conteúdo, aos fatores identificados na escala original canadense (relações com a equipe, maneira de ser tratado pelos profissionais e satisfação com as condições físicas do serviço). Houve uma ligeira diferença na distribuição dos itens entre os fatores, talvez por causa de diferenças culturais, características clínicas diferenciadas da população-alvo ou variações nas características dos serviços entre os dois países ${ }^{31}$. A ocorrência de distribuições diferenciadas dos itens nos fatores é frequente nas validações de escalas de medida de uma cultura original, a qual a escala foi inicialmente validada, para sua versão adaptada para um outro país ${ }^{14}$.

As correlações dos três fatores entre si e com a escala global mostraram que todas elas foram positivas e significativas. Além disso, como esperado, os valores dos coeficientes de correlação de cada fator com a escala global foram mais elevados do que os valores obtidos entre os fatores. Esse resultado indica que, embora os fatores avaliem dimensões distintas da satisfação com os serviços, eles compartilham um construto comum, referente à satisfação global dos pacientes com os serviços ${ }^{14}$. Não é possível fazer uma comparação desses resultados com os dados da escala original canadense, tendo em vista que esse tipo de análise não constava do estudo canadense $e^{31}$.

A consistência interna da escala SATIS-BR se mostrou adequada, com um valor de alfa de Cronbach igual a 0,88 para os itens da escala global. Esse resultado se assemelha ao obtido na escala original canadense, cujo valor de alfa foi igual a $0,89^{31}$. O valor de alfa obtido no presente estudo indica que os itens da escala global possuem consistência interna e se situam dentro da faixa considerada ideal por Vallerand ${ }^{14}$, entre 0,75 e 0,85, indicando que eles são homogêneos, sem ser redundantes. Os valores de alfa para os fatores 1 e 3 se situaram acima de 0,70. Apenas o fator 2 apresentou valor de alfa um pouco abaixo desse valor, porém ainda acima de 0,50, o que é considerado aceitáve ${ }^{32}$. As correlações item-total dos itens de cada fator variaram entre 0,36 e 0,89, portanto todas acima de 0,20, critério mínimo adotado para retenção dos 
itens na escala ${ }^{12}$. Esses valores foram superiores aos obtidos para a escala original, que se situaram entre 0,25 e 0,6831.

Quanto à validade convergente, avaliada pelo método da Análise por Hipótese ${ }^{29}$, a correlação positiva significativa obtida entre a escala SATIS-BR e a escala EMP $(r=0,41$; $p<0,001)$ confirmou a hipótese postulada de uma relação teórica entre os construtos de mudança percebida pelos próprios pacientes e de satisfação com o serviço. O valor da correlação entre as duas escalas foi moderado, tal como esperado, uma vez que se trata de medidas de dois construtos distintos, embora teoricamente relacionados. Este resultado comprova a validade convergente da escala SATIS-BR, uma vez que a escala foi capaz de comprovar a hipótese testada. A relação das mudanças percebidas pelos pacientes com sua satisfação em relação ao tratamento recebido nos serviços tem sido evidenciada por estudos desenvolvidos nos últimos anos, tais como as pesquisas de Hasler et al. ${ }^{7,33}$ e de Perreault et al. ${ }^{24}$.

\section{CONCLUSÃO}

Pode-se concluir que a escala SATIS-BR possui propriedades psicométricas adequadas de validade de construto, validade convergente e fidedignidade, para sua aplicação no contexto brasileiro, podendo ser utilizada para avaliar o grau de satisfação do pacientes com os serviços de saúde mental. Sendo uma escala multidimensional, ela é mais sensível para avaliar dimensões distintas dos serviços, o que possibilita obter resultados diferenciados em função de cada dimensão avaliada, contrariamente às escalas unifatoriais, que apresentam menor sensibilidade e menor grau de informações sobre a satisfação dos pacientes com os serviços ${ }^{6}$.

A escala permite identificar quais aspectos dos serviços são mais apreciados pelos pacientes ou, ao contrário, quais aspectos deixam a desejar e necessitam ser melhorados. Pode-se, assim, utilizar essa informação para redirecionar e aprimorar os serviços, visando melhorar sua qualidade. Os dados obtidos com a escala SATIS-BR poderão ser úteis para complementar as avaliações dos profissionais e ajudar o serviço a oferecer tratamentos mais adequados às necessidades dos pacientes. Tendo em vista a sua forma curta e a facilidade de aplicação e de compreensão da escala pela população-alvo, ela poderia ser aplicada em serviços de saúde mental, de forma rotineira ${ }^{10}$, visando incentivar uma cultura de avaliação de serviços de saúde mental e práticas baseadas em evidências ${ }^{34}$.

\section{AGRADECIMENTO}

Este artigo faz parte de um projeto de pesquisa financiado pela Fundação de Amparo à Pesquisa do Estado de Minas Gerais (Fapemig) e Conselho Nacional de Desenvolvimento
Científico e Tecnológico (CNPq) (site do LAPSAM: www.ufsj. edu.br/lapsam).

Agências financiadoras: Fapemig e CNPq. Conflito de interesses: nenhum.

\section{REFERÊNCIAS}

1. Organização Mundial de Saúde. Relatório Mundial da Saúde. Saúde mental: nova concepção, nova esperança. Lisboa: Divisão de Saúde Mental da OMS; 2001.

2. Donabedian A. Quality assurance in heath care: consumer's role. Qual Health Care. 1992;1:247-51.

3. Holcomb WR, Parker JC, Leong G, Thiele J, Higdon J. Customer satisfaction and self-reported treatment outcomes among psychiatric inpatients. Psychiatr Serv. 1998;49(7):929-34.

4. Einsen SV. Patient satisfaction and perceptions of care. In: IsHak WW, Burt T, Sederer LI, editors. Outcome measurement in psychiatry: a critical review. Washington: American Psychiatric Publishing; 2010. p. 303-20.

5. Ruggeri M. Satisfaction with mental health services. In: Thornicroft G, Tansella M, editors. Mental Health Outcome measures. London: RCPsych Publications; 2010. p. 99-115.

6. Ruggeri M. Patients' and relatives' satisfaction with psychiatric services: the state of the art of its measurement. Soc Psychiatry Psychiatr Epidemiol. 1994;29:212-27.

7. Hasler G, Moergeli H, Bachmann R, Lambreva E, Buddeberg C, Schnyder U. Patient satisfactions with outpatient psychiatric treatment: the role of diagnosis, pharmacotherapy, and perceived therapeutic change. Can J Psychiatry. 2004;49(5):315-21.

8. Hansson L. Outcome assessment in psychiatric service evaluation. Soc Psychiatry Psychiatr Epidemiol. 2001;36:244-8.

9. Priebe S, Gruyters T. Patients' assessment of treatment predicting outcome. Schizophr Bull. 1995;21:87-94.

10. Hunter R, Cameron R. The Scottish Schizophrenia Outcomes Study: a new paradigm for utilizing self-report assessments from patients with schizophrenia. Eur Psychiatr Rev. 2008; $1(2): 7-9$

11. Tait $L$, Lester $H$. Encouraging user involvement in mental health services. Adv Psychiatr Treat. 2005;11:168-75.

12. Perreault M, Katerelos TE, Sabourin E, Leichner P, Desmarais J. Information as a distinct dimension for satisfaction assessment of outpatient psychiatric services. Int J Health Care Qual Assur Inc Leadersh Health Serv. 2001;14(2-3):111-20.

13. Pascoe GC. Patient satisfaction in primary health care: a literature review and analysis. Eval Program Plann. 1983;6:185-210.

14. Vallerand RJ. Vers une méthodologie de validation trans-culturelle de questionnaires psychologiques: implications pour la recherché en langue française. Can Psychol. 1989;30(4):662-80.

15. Contandriopoulos AP, Champagne F, Denis JL, Pineault R. A avaliação na área de saúde: conceitos e métodos. In: Hartz ZMA, editor. Avaliação em saúde: dos modelos conceituais à prática na análise de implantação de programas. Rio de Janeiro: Editora Ficruz; 1997. p. 29-47.

16. Grogan $S$, Conner M, Norman P, Willits D, Porter I. Validation of a questionnaire measuring patient satisfaction with general practitioner services. Qual Health Care. 2000;9:210-5.

17. Bandeira M, Pitta AMF, Mercier C. Escalas da OMS de avaliação da satisfação e da sobrecarga em serviços de saúde mental: qualidades psicométricas da versão brasileira. J Bras Psiquiatr. 1999;48(6):233-44.

18. Bandeira M, Pitta AMF, Mercier C. Escala de avaliação da satisfação dos usuários em serviços de saúde mental: SATIS-BR. J Bras Psiquiatr. 2000;49(8):293-300.

19. Artes R. Aspectos estatísticos da análise fatorial de escalas de avaliação. Rev Psiq Clín. 1998;25(5):223-8.

20. Organização Mundial de Saúde. CID-10: Classificação Internacional de Doenças e problemas relacionados à saúde. São Paulo: EDUSP; 1998.

21. World Health Organization. WHO-SATIS consumer's and caregivers' satisfaction with mental health services a multisite study. Geneva: Mental Health World Organization; 1996. 
22. Larsen DL, Attkisson CC, Hargreaves WA, Nguyen TD. Assessment of client/patient satisfaction: development of a general scale. Eval Program Plann. 1979;2:197-207.

23. Mercier L, Landry M, Corbière M, Perreault M. Measuring clients perception as outcome measurement. In: Roberts AR, Yeager KR, editors. Evidence-based practice manual: research an outcome measures in health and human services. New York: Oxford University Press; 2004. p. 904-9.

24. Perreault M, White ND, Fabrès E, Landry M, Anestin AS, Rabouin D. Relationship between perceived improvement and treatment satisfaction among clients of a methadone maintenance program. Eval Program Plann. 2010;33(4):410-7.

25. Bandeira M, Calzavara MGP, Costa CS, Cesari L. Avaliação de serviços de saúde mental: adaptação transcultural de uma medida da percepção dos usuários sobre os resultados do tratamento. J Bras Psiquiatr. 2009;58(2):107-14.

26. Bandeira M, Andrade MCR, Costa CS, Silva MA. Percepção dos pacientes sobre o tratamento em serviços de saúde mental: validação da Escala de Mudança Percebida. Psicologia: Reflexão e Crítica. 2011;24(2):236-44

27. Dancey CP, Reidy J. Estatística sem matemática para psicologia. Usando SPSS para Windows. Porto Alegre: Artmed; 2006.
28. Zwick WR, Velicer WF. Comparison of five rules for determining the number of components to retain. Psychol Bull. 1986;99(3):432-42.

29. Pasquali L. Validade dos testes. In: Pasquali L, editor. Psicometria: teoria dos testes na Psicologia e na Educação. Petrópolis, RJ: Vozes; 2003. p. 158-88.

30. Likert R. A technique for the measurement of attitudes. Arch Psychol. 1932;140:1-55

31. St-Onge M, Mercier C, Perreault M, Harnois G. La mesure de la satisfaction et du fardeau dans le domaine de la santé mentale. Résultats de l'enquete quebecoise de validation des echelles de I OMS. Montreal (CA): Centre Collaborateur OMS de Montréal, Centre de recherche de l'Hôpital Douglas; 1997.

32. Bowling A. Measuring disease: a review of disease specific quality of life measurement scales. Philadelphia: Open University Press; 1997.

33. Hasler G, Moergeli H, Schnyder U. Outcome of psychiatric treatment: what is relevant for our patients? Compr Psychiatry. 2004;45(3):199-205.

34. Thornicroft $\mathrm{G}$, Tansella M. Saúde mental da população e cuidado comunitário. In: Thornicroft G, Tansella M, editores. Boas práticas em saúde mental comunitária. Barueri, SP: Manole; 2010. p. 6-19.

\section{ESCALA DE AVALIAÇÃO DA SATISFAÇÃO DOS PACIENTES COM OS SERVIÇOS DE SAÚDE MENTAL}

\begin{tabular}{|l|l|}
\hline Nome (Opcional): & Idade: \\
\hline Estado civil: & Escolaridade: \\
\hline Sexo: & Trabalho: \\
\hline Nome do serviço: & Início do atendimento: \\
\hline
\end{tabular}

Data

Nós vamos Ihe fazer algumas perguntas sobre o seu grau de satisfação geral com o (nome do serviço). Eu vou ler para você todas as perguntas e todos os tipos de resposta. Não há respostas certas ou erradas. Queira responder de acordo com sua opinião pessoal.

1. Qual a sua opinião sobre a maneira como você foi tratado, em termos de respeito e dignidade?

\begin{tabular}{|l|l|}
\hline Nunca me senti respeitado & 1 \\
\hline Geralmente não me senti respeitado & 2 \\
\hline Mais ou menos & 3 \\
\hline Geralmente me senti respeitado & 4 \\
\hline Sempre me senti respeitado & 5 \\
\hline
\end{tabular}

2. Quando você falou com a pessoa que admitiu você no (nome do serviço), você sentiu que ele/a ouviu você?

\begin{tabular}{|l|c|}
\hline Não me ouviu de forma alguma & 1 \\
\hline Não me ouviu bastante & 2 \\
\hline Mais ou menos & 3 \\
\hline Me ouviu bastante & 4 \\
\hline Me ouviu muito & 5 \\
\hline
\end{tabular}

3. Até que ponto a pessoa que admitiu você no (nome do serviço) pareceu compreender o seu problema?

\begin{tabular}{|l|c|}
\hline Não me compreendeu de forma alguma & 1 \\
\hline Não me compreendeu muito & 2 \\
\hline Mais ou menos & 3 \\
\hline Me compreendeu bem & 4 \\
\hline Me compreendeu muito bem & 5 \\
\hline
\end{tabular}

4. Em geral, como você acha que a equipe do (nome do serviço) compreendeu o tipo de ajuda de que você necessitava?

\begin{tabular}{|l|c|}
\hline Não me compreendeu de forma alguma & 1 \\
\hline Não me compreendeu muito & 2 \\
\hline Mais ou menos & 3 \\
\hline Me compreendeu bem & 4 \\
\hline Me compreendeu muito & 5 \\
\hline
\end{tabular}

5. Qual sua opinião sobre o tipo de ajuda dada a você pelo (nome do serviço)?

\begin{tabular}{|l|c|}
\hline Parece que eles pioraram as coisas & 1 \\
\hline Não obtive nenhuma ajuda & 2 \\
\hline Não obtive muita ajuda & 3 \\
\hline Senti que obtive alguma ajuda & 4 \\
\hline Senti que obtive muita ajuda & 5 \\
\hline
\end{tabular}

6. Até que ponto você está satisfeito com a discussão que foi feita com você sobre o seu tratamento no (nome do serviço)?

\begin{tabular}{|l|c|}
\hline Muito insatisfeito & 1 \\
\hline Insatisfeito & 2 \\
\hline Indiferente & 3 \\
\hline Satisfeito & 4 \\
\hline Muito satisfeito & 5 \\
\hline
\end{tabular}


7. Você considerou que a equipe do (nome do serviço) estava Ihe ajudando?

\begin{tabular}{|l|c|}
\hline Nunca & 1 \\
\hline Raramente & 2 \\
\hline Mais ou menos & 3 \\
\hline Frequentemente & 4 \\
\hline Sempre & 5 \\
\hline
\end{tabular}

8. Em geral, como você classificaria a acolhida dos profissionais do (nome do serviço)?

\begin{tabular}{|l|c|}
\hline Nada amigável & 1 \\
\hline Pouco amigável & 2 \\
\hline Mais ou menos & 3 \\
\hline Amigável & 4 \\
\hline Muito amigável & 5 \\
\hline
\end{tabular}

9. Em geral, como você classificaria a competência da equipe do (nome do serviço)?

\begin{tabular}{|l|c|}
\hline Muito incompetente & 1 \\
\hline Incompetente & 2 \\
\hline Mais ou menos & 3 \\
\hline Competente & 4 \\
\hline Muito competente & 5 \\
\hline
\end{tabular}

10. Na sua opinião, que grau de competência tinha a pessoa com quem você trabalhou mais de perto?

\begin{tabular}{|l|c|}
\hline Muito incompetente & 1 \\
\hline Incompetente & 2 \\
\hline Mais ou menos & 3 \\
\hline Competente & 4 \\
\hline Muito competente & 5 \\
\hline
\end{tabular}

11. Você ficou satisfeito com o conforto e a aparência do (nome do serviço)?

\begin{tabular}{|l|c|}
\hline Muito insatisfeito & 1 \\
\hline Insatisfeito & 2 \\
\hline Indiferente & 3 \\
\hline Satisfeito & 4 \\
\hline Muito satisfeito & 5 \\
\hline
\end{tabular}

12. Como você classificaria as condições gerais das instalações (p. ex., instalações de banheiro/cozinha, refeições, prédio, etc.)?

\begin{tabular}{|l|c|}
\hline Péssimas & 1 \\
\hline Ruins & 2 \\
\hline Regulares & 3 \\
\hline Boas & 4 \\
\hline Excelentes & 5 \\
\hline
\end{tabular}

13. De que você mais gostou no (nome do serviço)?

14. De que você menos gostou no (nome do serviço)?

15. Na sua opinião, o serviço no (nome do serviço) poderia ser melhorado?

\begin{tabular}{|l|c|}
\hline Sim & 1 \\
\hline Não & 2 \\
\hline Não sei & 3 \\
\hline
\end{tabular}

\subsection{Se sim, de que maneira?}

OBRIGADO PELA SUA PARTICIPAÇÃO!!! 